\title{
Neutrino physics with the SHiP experiment at CERN
}

\section{De Serio*i}

Dipartimento di Fisica Università di Bari and I.N.F.N. Bari, via Amendola 173, Bari, Italy E-mail: marilisa.deserio@ba.infn.it

SHiP (Search for Hidden Particles) is a beam-dump experiment at CERN SPS conceived to search for long-lived exotic particles with masses below a few $\mathrm{GeV} / \mathrm{c}^{2}$, produced in the decays of heavy hadrons. The abundant production of neutrinos in such processes makes SHiP also ideally suited for $v$ physics studies. A dedicated detector, consisting of a target made of nuclear emulsion - lead bricks alternating with planes of electronic trackers, and a muon spectrometer, has been designed to provide the first direct observation of the $\bar{v}_{\tau}$ and measure the $v_{\tau}$ and $\bar{v}_{\tau}$ cross-sections.

EPS-HEP 2017, European Physical Society conference on High Energy Physics 5-12 July 2017

Venice, Italy

* Speaker.

On behalf of the SHiP Collaboration. 


\section{Physics motivation}

The Standard Model (SM) of particle physics is able to explain a wide variety of experimental results, thus providing a consistent description of Nature's fundamental constituents and their interactions. Nevertheless, it fails in accounting for some observed phenomena, such as the masses and oscillations of neutrinos, the excess of matter over antimatter in the Universe and the existence of dark matter. In order to embed these unaccounted experimental evidences in the theoretical framework, several models predict the existence of long-lived neutral particles, with very weak couplings to SM particles, that could be hiding in the largely unexplored domain at the so-called intensity frontier.

SHIP [1], [2] is a fixed-target experiment at CERN SPS designed to look for long-lived hidden particles with masses below a few $\mathrm{GeV} / \mathrm{c}^{2}$, produced in the decays of beauty and charm mesons (e.g. heavy neutral leptons, HNLs [3]). A $400 \mathrm{GeV} / \mathrm{c}$ proton beam extracted from the SPS will be dumped on a molybdenum-tungsten target maximising the production of heavy hadrons thanks to its short interaction length. Since a high flux of neutrinos of all flavours is expected from the decays of such heavy mesons, SHiP will be also able to perform neutrino physics studies, such as the first observation of the $\bar{v}_{\tau}$ and the measurement of $v_{\tau}$ and $\bar{v}_{\tau}$ cross-sections with unprecedented statistics.

\section{The SHiP detector}

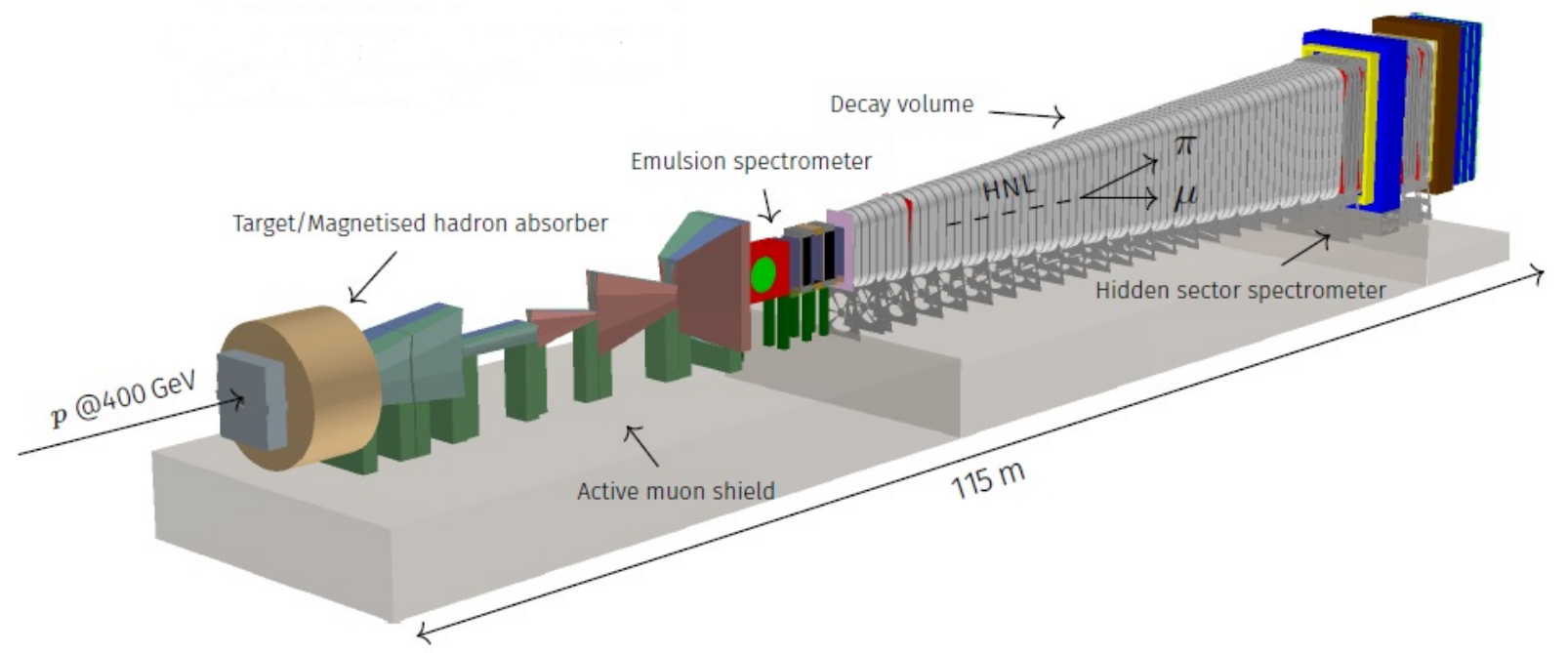

Figure 1: Layout of the SHiP detector.

Figure 1 shows the current layout of the SHiP detector. It consists of a $v$ detector and a Hidden Particles (HP) detector, located downstream of a hadron stopper and an active muon shield, designed to bend out beam-related muons through magnetic deflection [4]. 


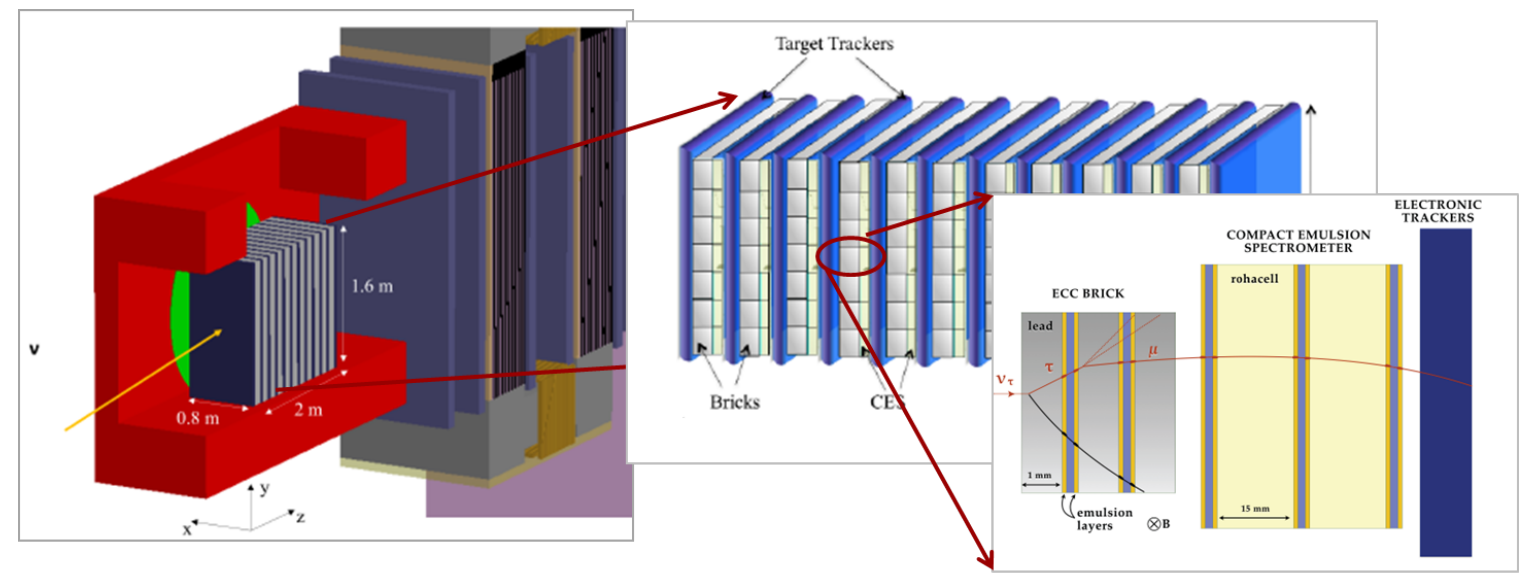

Figure 2: Layout of the $v$ detector with magnetised target.

The $v$ detector consists of a compact high-density target, followed by a muon magnetic spectrometer. The target has a modular structure. It consists of walls of ECC (Emulsion Cloud Chamber) bricks, similar to those exploited in the OPERA experiment [5], namely plates of high-Z passive material (e.g. lead) interleaved with thin nuclear emulsion foils acting as sub-micrometric position resolution detectors, as required to disentangle the $v_{\tau}$ interaction and the $\tau$ lepton decay vertices. Electronic trackers, providing event time stamping, are located in between target walls and allow connecting the tracks reconstructed in the emulsion target with those measured in the downstream spectrometer. This layout has already proven to be effective in detecting all $v$ flavors $[6,7,8,9,10,11]$. The separation of $v_{\tau} / \bar{v}_{\tau}$ through the decay of the $\tau$ lepton in the muon channel can be performed by measuring the electric charge of the muon in the spectrometer. The capabilities of the $v$ detector can be further extended by using a magnetised target which would allow to separate $v_{\tau} V_{\tau}$ also through the decay of the $\tau$ lepton in the hadronic channel. This layout, illustrated in figure 2, foresees the use of a magnetic field of $\sim 1 \mathrm{~T}$ and of a compact emulsion spectrometer (CES), made of a sequence of very low density material and emulsion foils, designed to measure the electric charge and momentum of the hadrons through their curvature in the magnetic field.

The HP detector, located downstream of the $v$ detector, consists of a long evacuated vessel, acting as a decay tunnel for possible exotic neutral particles with $\mathrm{c} \tau \sim O(\mathrm{~km})$, instrumented with a veto system and followed by detectors with particle identification and timing capabilities. Details on the HP detector and physics can be found in the paper by O. Lantwin in these proceedings.

\section{Neutrino physics performance}

The search for hidden particles requires an abundant production of heavy hadrons whose decays produce, in turn, a large flux of neutrinos. The expected flux of tau neutrinos, copiously produced via $D_{s} \rightarrow \tau \nu_{\tau}$ and subsequent $\tau \rightarrow \nu_{\tau}$ decay, is particularly high as compared to previous experiments, since the proton beam and target have been specifically optimised to enhance the production of charmed mesons and of Ds mesons. So far, only $O(10) v_{\tau}$ s have been observed in the 
Table 1: Expected rates of $v$ CC DIS interactions for $2 \times 10^{20}$ p.o.t. and a neutrino target mass of 7 tons.

\begin{tabular}{ccc}
\hline $\begin{array}{c}\text { Neutrino } \\
\text { flavor }\end{array}$ & $\begin{array}{c}<\mathrm{E}> \\
(\mathrm{GeV})\end{array}$ & $\begin{array}{c}\mathrm{CC} \\
\text { DIS }\end{array}$ \\
\hline$v_{\mu}$ & 31 & $2.3 \times 10^{6}$ \\
$v_{e}$ & 50 & $6.3 \times 10^{5}$ \\
$v_{\tau}$ & 45 & $2.6 \times 10^{4}$ \\
$\bar{v}_{\mu}$ & 26 & $8.6 \times 10^{5}$ \\
$\bar{v}_{e}$ & 38 & $2.2 \times 10^{5}$ \\
$\bar{v}_{\tau}$ & 58 & $1.9 \times 10^{4}$ \\
\hline
\end{tabular}

Table 2: Expected $v$-induced charm yield for $2 \times 10^{20}$ p.o.t. and a neutrino target mass of 7 tons.

\begin{tabular}{ccc}
\hline $\begin{array}{c}\text { Neutrino } \\
\text { flavor }\end{array}$ & $\begin{array}{c}<\mathrm{E}> \\
(\mathrm{GeV})\end{array}$ & $\begin{array}{c}\text { Charm } \\
\text { events }\end{array}$ \\
\hline$v_{\mu}$ & 48 & $1.0 \times 10^{5}$ \\
$v_{e}$ & 59 & $3.9 \times 10^{4}$ \\
$\bar{v}_{\mu}$ & 41 & $3.6 \times 10^{4}$ \\
$\bar{v}_{e}$ & 49 & $1.2 \times 10^{4}$ \\
\hline
\end{tabular}

DONUT [12] and OPERA [6, 7, 8, 9, 10] experiments. This makes the $v_{\tau}$ the less known fermion in the SM. Moreover, the $\bar{v}_{\tau}$ has never been detected experimentally.

Table 1 shows the expected rates of $v$ CC DIS interactions assuming an integrated beam intensity of $2 \times 10^{20}$ p.o.t. in five years of data-taking and a neutrino target mass of 7 tons. The $v_{\tau} / \bar{v}_{\tau}$ statistics is a factor of $10^{3}$ larger than the overall statistics collected by previous experiments and will allow measuring the $v_{\tau} / \bar{v}_{\tau}$ cross-sections, as well as the structure functions $\mathrm{F}_{4}$ and $\mathrm{F}_{5}$ whose contributions to the $v_{\mu}$ and $v_{e}$ cross-sections are negligible.

Table 2 summarises the expected numbers of $v$-induced charm events in five years run. Since charmed hadrons have lifetimes and decay topologies similar to those of the $\tau$ lepton, they can be efficiently detected using an emulsion target. The overall yield exceeds the available statistics by more than one order of magnitude, thus making it possible to improve the accuracy of current measurements as well as to study unexplored channels. As an example, the production of charmed hadrons in $v_{e}$ interactions has never been reported so far.

The production of charmed particles in $\bar{v}$ interactions occurs through the scattering of the incoming $v$ off a $\bar{d}$ or $\bar{s}$ quark. Since $\mathrm{d} \rightarrow \mathrm{c}$ quark transitions are Cabibbo-suppressed, $\bar{v}$-induced charm production allows probing the strange quark content of the nucleon. Defining $s^{ \pm}=s(x) \pm$ $\bar{s}(x)$, an improvement in the accuracy of almost a factor of two is expected in the range of the Bjorken variable $\mathrm{x}$ between 0.03 and 0.35 for $s^{+}$and between 0.08 and 0.30 for $s^{-}$. 


\section{Conclusions and Outlook}

The SHiP experiment has been designed to probe the intensity frontier by searching for new, very weakly interacting particles with masses $O\left(\mathrm{GeV} / \mathrm{c}^{2}\right)$. A rich physics program, including the search for Heavy Neutral Leptons and $v$ physics studies with unprecedented statistics, is foreseen. Currently, the experiment is in the Comprehensive Design phase aiming at the European Strategy meeting in 2019.

\section{References}

[1] M. Anelli et al. (SHiP Collaboration), A facility to Search for Hidden Particles (SHiP) at the CERN SPS, CERN-SPSC-2015-016, SPSC-P-350, arXiv:1504.04956, 2015

[2] S. Alekhin et al., A facility to Search for Hidden Particles at the CERN SPS: the SHiP physics case, CERN-SPSC-2015-017, arXiv:1504.04855, 2015

[3] T. Asaka and M. Shaposhnikov, The VMSM, dark matter and baryon asymmetry of the Universe, Phys. Lett. B 620 17, 2005

[4] A. Akmete et al. (SHiP Collaboration), The active muon shield in the SHiP experiment, JINST 12 n.05, P05011, 2017

[5] R. Acquafredda et al. (OPERA Collaboration), The OPERA experiment in the CERN to Gran Sasso neutrino beam, JINST 4 P04018, 2009

[6] N. Agafonova et al. (OPERA Collaboration), Observation of a first $v_{\tau}$ candidate in the OPERA experiment in the CNGS beam, Phys. Lett. B 691 138, 2010

[7] N. Agafonova et al. (OPERA Collaboration), New results on $v_{\mu} \rightarrow v_{\tau}$ appearance with the OPERA experiment in the CNGS beam, JHEP 11 036, 2013

[8] N. Agafonova et al. (OPERA Collaboration), Evidence for $v_{\mu} \rightarrow v_{\tau}$ appearance in the CNGS neutrino beam with the OPERA experiment, Phys. Rev. D 89 051102, 2013

[9] N. Agafonova et al. (OPERA Collaboration), Observation of tau neutrino appearance in the CNGS beam with the OPERA experiment, PTEP 101C01, 2014

[10] N. Agafonova et al. (OPERA Collaboration), Discovery of tau neutrino appearance in the CNGS neutrino beam with the OPERA experiment, Phys. Rev. Lett. 115 121802, 2015

[11] N. Agafonova et al. (OPERA Collaboration), Search for $v_{\mu} \rightarrow v_{e}$ oscillations with the OPERA experiment in the CNGS beam, JHEP 1307 004, 2013

[12] K. Kodama et al. (DONUT Collaboration), Final tau-neutrino results from the DONuT experiment, Phys. Rev. D 78 052002, 2008 